\title{
Erratum to: Protective Effects of Radon Inhalation on Carrageenan-Induced Inflammatory Paw Edema in Mice
}

\author{
Takahiro Kataoka, ${ }^{1}$ Junichi Teraoka, ${ }^{1}$ Akihiro Sakoda, ${ }^{2}$ Yuichi Nishiyama, ${ }^{1}$ Keiko Yamato, ${ }^{1}$ \\ Mayuko Monden, ${ }^{3}$ Yuu Ishimori, ${ }^{2}$ Takaharu Nomura, ${ }^{4}$ Takehito Taguchi, ${ }^{1}$ and \\ Kiyonori Yamaoka ${ }^{1,5}$
}

\section{Erratum to: Inflammation \\ DOI 10.1007/s10753-011-9364-y}

The original version of this article unfortunately contained a mistake. The Y-axis label in upper left side of the Fig. $3 \mathrm{~d}$ is in error and should be changed as follows:

The online version of the original article can be found at http:// dx.doi.org/10.1007/s10753-011-9364-y.

\footnotetext{
${ }^{1}$ Graduate School of Health Sciences, Okayama University, 5-1 Shikatacho 2-chome, Kita-ku, Okayama-shi, Okayama 700-8558, Japan

${ }^{2}$ Ningyo-toge Environmental Engineering Center, Japan Atomic Energy Agency, 1550 Kagamino-cho, Tomata-gun, Okayama 708-0698, Japan ${ }^{3}$ Department of Pharmacology, Faculty of Pharmacy and Pharmaceutical, Sciences, Fukuyama University, 1-Gakuen-cho, Fukuyama-shi, Hiroshima 729-0292, Japan

${ }^{4}$ Radiation Safety Research Center, Central Research Institute of Electric Power Industry, 2-11-1 Iwado-kita, Komae-shi, Tokyo 201-8511, Japan ${ }^{5}$ To whom correspondence should be addressed at Graduate School of Health Sciences, Okayama University, 5-1 Shikata-cho 2-chome, Kita-ku, Okayama-shi, Okayama 700-8558, Japan. E-mail: yamaoka@md. okayama-u.ac.jp
}
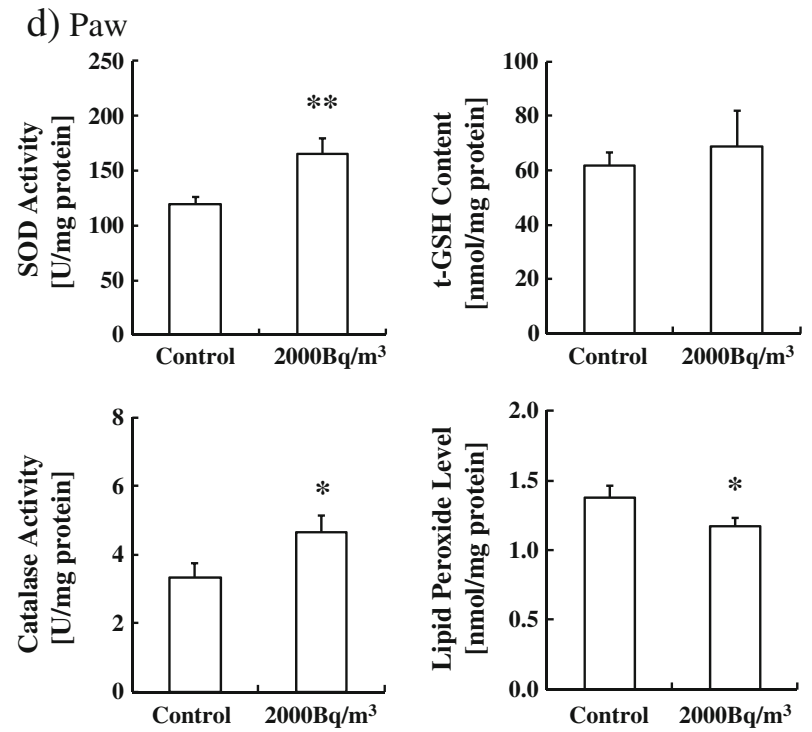

The Y-axis label in upper left side of the Fig. 3d should be "SOD Activity [U/mg protein]". 